\title{
Design and Evaluation of Broad-Band High Pass Filter of Metal Perforated Sheets for Microwave Diagnostics ${ }^{*}$
}

\author{
Akira TANI, Hayato TSUCHIYA ${ }^{1)}$, Mayuko KOGA and Yoshio NAGAYAMA ${ }^{1)}$ \\ Graduate School of Engineering, University of Hyogo, 2167 Shosha, Himeji 671-2280, Japan \\ 1) National Institute for Fusion Science, 322-6 Oroshi-cho, Toki 509-5292, Japan
}

(Received 27 November 2015 / Accepted 3 February 2016)

\begin{abstract}
This paper reports characteristic evaluations of perforated metal sheet type high pass filters for milli- or microwave by numerical analysis and the guiding principle of designing broad-band high pass filters. The broadband filters will be used for the microwave plasma diagnostics such as ECE, interferometer and reflectometer. We used 3D-modeling electromagnetic field calculation code to take complex interferences into account. The validity of the numerical analysis is guaranteed by a good agreement between calculated results and measured results. It is found that the frequency characteristic over the cut-off frequency depends on the filter thickness and that a thin filter is desirable for the ideal broad-band frequency filter.
\end{abstract}

(C) 2016 The Japan Society of Plasma Science and Nuclear Fusion Research

Keywords: ECE, microwave diagnostics, millimetric-wave, perforated metal sheet, broad-band high pass filter, $3 \mathrm{D}$ numerical analysis, Efield

DOI: $10.1585 /$ pfr. 11.2405027

\section{Introduction}

Microwave diagnostics in plasma experiment are popular techniques in both large and small size plasma devices. The frequency band of microwave used for this diagnostics generally depends on the plasma density and the magnetic field strength. In the case of fusion oriented plasma experiment devices, the typical wavelength is millimeter to submillimeter. Recently, most diagnostics require the multichannel systems or 2D/3D imaging systems for further plasma physical study and plasma control. To realize such advanced diagnostics, development of microwave components are necessary. In particular, ultra-narrow notch filters and broad band high/low pass filters are often unique. In designing an imaging diagnostic, the power density profile as well as the frequency characteristics of the filter must be taken into account.

Diagnostics of Electron Cyclotron Emission (ECE), which is caused by electron cyclotron motions in magnetically confined plasma, are major multi-channel devices, and they are often applied for imaging diagnostics. As the radiation intensity of the second harmonic X-mode of ECE is proportional to an electron temperature, the emission location can be identified by its frequency. For this reason, ECE measurement is a useful method in observation of electron temperature profiles and their local fluctuations. It is possible to measure the broad-band ECE by Michelson interferometer for obtaining the radial temperature profile. For measuring electron temperature fluctuations, a heterodyne-type radiometer is generally used. The

author'se-mail:er15e007@steng.u-hyogo.ac.jp

*) This article is based on the presentation at the 25th International Toki Conference (ITC25). frequency band of ECE in high magnetic field devices is broader than that of the radiometer. Therefore, two radiometer systems with different local frequency are used to detect broad-band ECE in high-field devices. It is necessary to separate the RF with high precision before two systems of heterodyne detection. In the case of the Large Helical Device (LHD), the frequency range of ECE is 50 $150 \mathrm{GHz}[1,2]$. In a future device such as ITER the frequency band will become higher. At present, the broadband high pass filter for such frequency (E-band, W-band) is not commercially available. Therefore, we have developed an original broad-band high pass filter. The filter can be also used for c-ECE (correlation ECE) with second heterodyne and microwave imaging reflectometry [3].

The purpose of this study is to design perforated metal sheet type broad-band filters (cut-off frequency is $44 \mathrm{GHz}$ ). We designed broad-band high pass filters and evaluated their characteristics including effects of complex interferences by 3D-numerical electromagnetic field analysis.

\section{Analytic Solution of Perforated Metal Sheet Filter}

The schematic of the perforated metal sheet filter is shown in Fig. 1. It is possible to regard the perforated metal sheet (filter) with circular holes as an ensemble of short waveguides whose length corresponds to the filter thickness. We focused on the property of electromagnetic waves propagating in waveguides. It is, in principle, assumed that higher waves than cut-off frequency $\left(f_{\mathrm{c}}\right)$ can transmit in waveguides and that lower waves than $f_{\mathrm{c}}$ are reflected. If we assume the propagating mode is only $\mathrm{TE}_{11}$ mode, which is the lowest-order mode of circular waveg- 


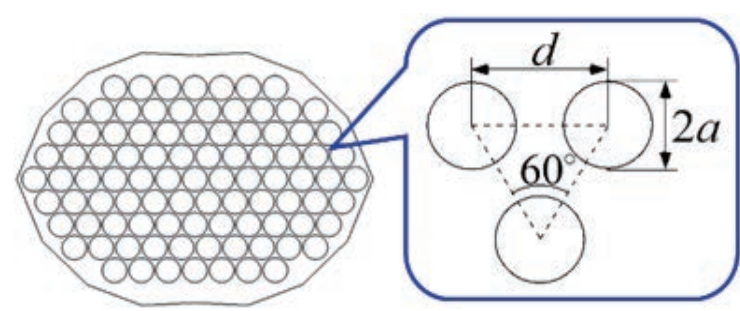

Fig. 1 Schematic of the metal perforated plate with circular holes. Holes are arranged periodically.

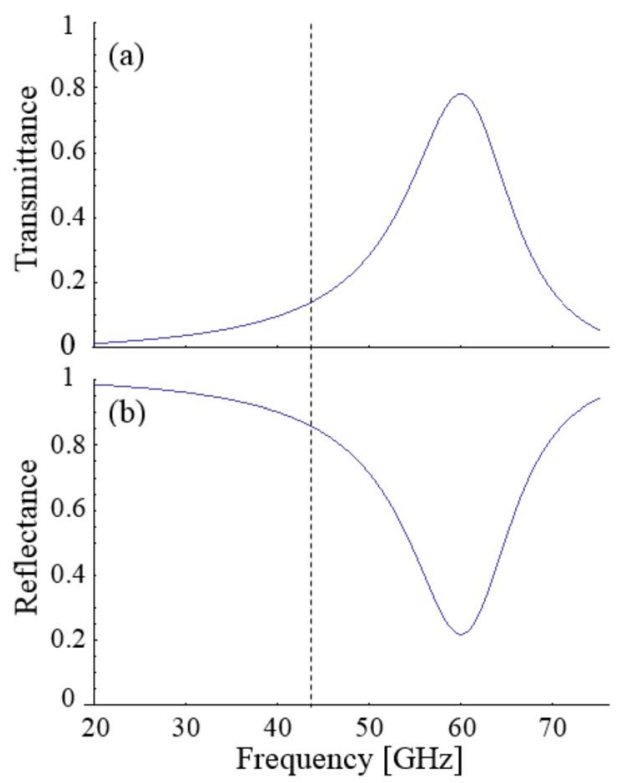

Fig. 2 Theoretical frequency characteristics. The dot-line indicates the cut-off frequency $(44 \mathrm{GHz})$. Filter thickness 1 is $1 \mathrm{~mm}$. The distance of holes $d$ is $4.3 \mathrm{~mm}$.

uide, radius $a$ is expressed by the following equation;

$$
a=\frac{1.841 c}{2 \pi f_{\mathrm{c}}}
$$

where $c$ is light speed $\left(3.0 \times 10^{11} \mathrm{~mm} / \mathrm{s}\right)$.

For the case of normal incidence, the transmission coefficients $(T)$ and the reflection coefficients $(R)$ of the filter can be reduced to the following forms [4].

$$
\begin{aligned}
T= & \frac{1}{1-j[A+B \tanh (\beta l)]} \\
& -\frac{1}{1-j[A+B \operatorname{coth}(\beta l)]}, \\
R= & \frac{1}{1-j[A+B \tanh (\beta l)]} \\
& +\frac{1}{1-j[A+B \operatorname{coth}(\beta l)]}-1,
\end{aligned}
$$

where and are functions of $a, d$ and wavelength $\lambda, \beta$ is a propagation constant, and $l$ is filter thickness (see Ref. 4 for more detail). Theoretical frequency characteristics in the condition calculated in the next section derived by above equations are shown in Fig. 2. The perpendicular polarization is assumed. The figures show that waves of higher

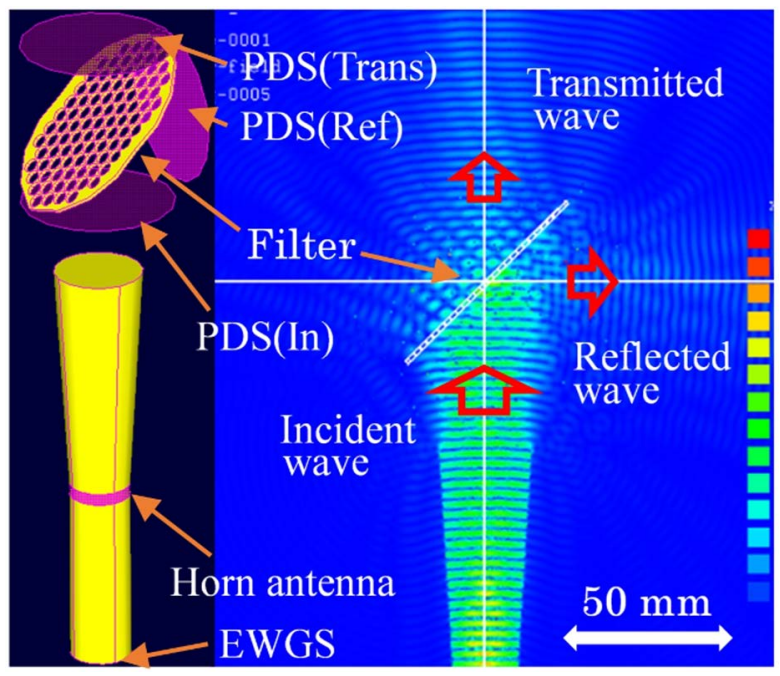

Fig. 3 (Left) Layout of filter and horn antenna. Waves are generated on the bottom surface of the wave guide and propagate through a coaxial wave guide and a horn antenna. (Right) Aspect of wave propagation near the tested filter. The color bar shows the amplitude of electric field. (PDS: Power Detection Surface, EWGS: Electromagnetic Wave Generation Surface).

frequency than $f_{\mathrm{c}}$ can transmit the filter, however, the characteristic in high frequency range has a peak, and the transmittance over $70 \mathrm{GHz}$ becomes small. It is necessary to improve the frequency characteristic for the broad-band high pass filter.

\section{3D-Numerical Analysis Model and Methods}

In this calculation, $f_{\mathrm{c}}$ is assumed to be $44 \mathrm{GHz}$. According to Eq. (1), $a$ is $2.0 \mathrm{~mm}$, and $d$ is $4.3 \mathrm{~mm}$, which is the closest distance with keeping a realistic manufacturing precision. Filter thicknesses are set to 4 patterns: $0 \mathrm{~mm}$, $0.5 \mathrm{~mm}, 1 \mathrm{~mm}$, and $2 \mathrm{~mm}$. Material of filters are perfect conductors. The used 3DCAD software is CADfix [5].

3D-numerical analysis is performed using Efield [6]. The analysis model is shown in Fig. 3. The tested filter is tilted at 45-degree angle to the incident direction of electromagnetic waves. Electromagnetic waves are generated on EWGS (Electromagnetic Wave Generation Surface) and are emitted to the filter from a horn antenna. A horn antenna is installed to improve the directionality of electromagnetic waves and to stabilize the propagation mode. The generated waves are monochromatic waves, and the frequency can be set arbitrarily. The incident wave power to the filter is calculated on the power detection surface (PDS (In)). The transmitted/reflected power surface (PDS (Trans/Ref)) are set just after the transmission/reflection on the axis, respectively. Transmittance and reflectance in every frequency are defined as the ratio of integrated power on PDS (Trans/Ref) to integrated power on PDS (In) in this calculation model. The mesh spacing is $0.4 \mathrm{~mm}$, which is 


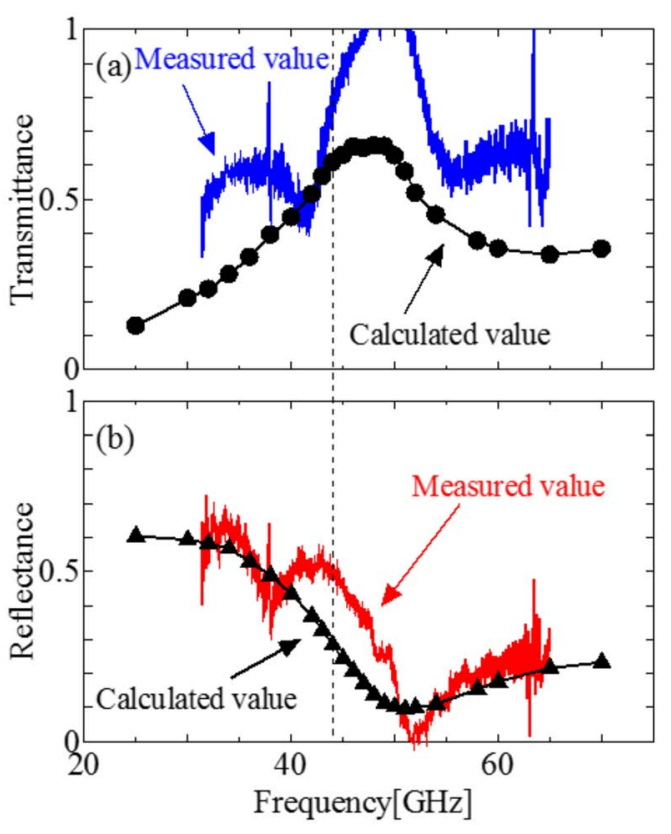

Fig. 4 The comparison of the calculated and the measured frequency characteristics. (a) Frequency dependence of transmittance (b) Frequency dependence of reflectance. The dot-line indicates cut-off frequency $(44 \mathrm{GHz})$. Filter thickness is $1 \mathrm{~mm}$.

less than one quarter of the wave length. The analytical algorithm is method of moment (MoM).

\section{Analysis Result}

The experiment of an actual filter has been performed in the same layout as the simulation model layout in Fig. 3. Frequency characteristics of an actual filter are investigated by using a vector network analyzer. Microwaves propagating through free space are measured by disposed a rectangular horn antenna.

Calculated and measured frequency characteristics are compared in Fig. 4. In calculation, we analyzed a filter characteristic in the range of $25-70 \mathrm{GHz}$. As frequency increases, the calculated transmittance increases up to about 0.7 around $48 \mathrm{GHz}$, and it decreases in high frequency range. The measured transmittance also has similar trends to the calculated filter characteristic. As frequency increases, the calculated reflectance reaches to the minimum around $52 \mathrm{GHz}$. The measured reflectance also has similar trends to the calculated filter characteristic. The reflectance profile and the absolute value of calculated characteristic are approximately equal to the measured. Considering that the measured characteristics may contain noises of measuring instruments, the calculated results are in good agreement with measured results. The theoretical characteristic of transmittance has peak, however, the theoretical peak frequency is different from the peak frequency that was both measured and calculated. These results indicate that our 3D-numerical simulation rather than the theoreti-
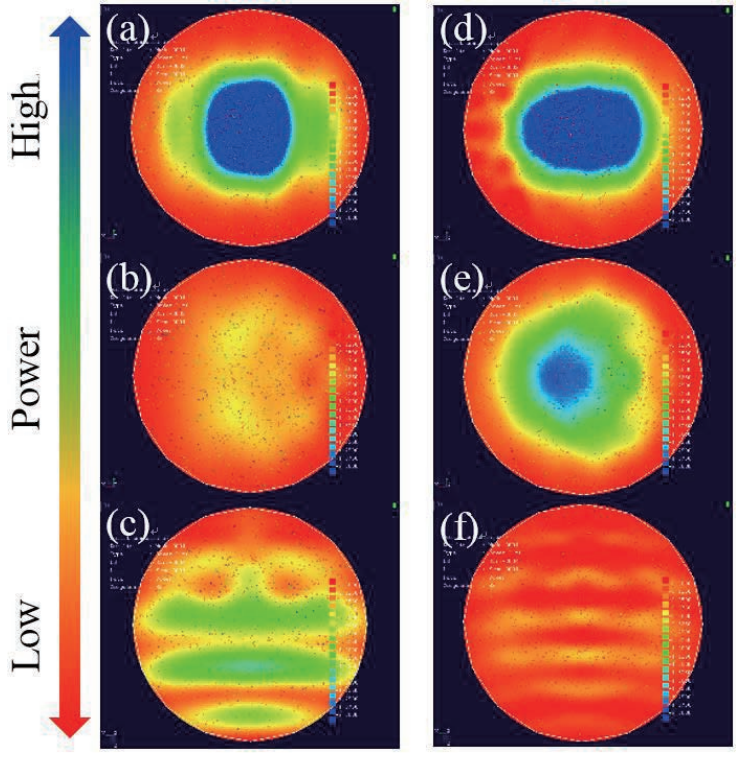

Fig. 5 Power profiles. (a) PDS of the incident wave in $34 \mathrm{GHz}$. (b) PDS of the transmitted wave in $34 \mathrm{GHz}$. (c) PDS of the reflected wave in $34 \mathrm{GHz}$. (d) PDS of the incident wave in $48 \mathrm{GHz}$. (e) PDS of the transmitted wave in $48 \mathrm{GHz}$. (f) PDS of the reflected wave in $48 \mathrm{GHz}$. The color bar shows the amplitude of power. The filter thickness is $1 \mathrm{~mm}$. The designed $\mathrm{fc}$ is $44 \mathrm{GHz}$.

cal solution can reconstruct faithfully the actual phenomena of microwave propagation. The detail trends of the calculated and the measured frequency characteristics in Fig. 4 are poorly-matched. Calculated values show only filter characteristics. However, measured values include characteristics of the antenna and the cable. We consider that these differences cause the poorly-matched result.

Power profiles of the incident wave, the reflectance wave and the transmittance wave (filter thickness is $1 \mathrm{~mm}$, cut-off frequency is $44 \mathrm{GHz}$ ) on PDS are shown in Fig. 5. The left column pictures show profiles in the case of a frequency lower than $f_{\mathrm{c}}$. The right column pictures are profiles of the higher frequency case. The incident wave power has high power peak in central of PDS (see. Figs. 5 (a), (d)), which indicates the emission of $\mathrm{TE}_{11}$ mode from a horn antenna. Next, when we compare the transmitted wave power profile, it is clearly found that the power intensity in the low frequency case (Fig. 5 (b)) is lower than that in the high frequency case, and the transmittance wave power profile does not change significantly from the incident wave power profile. The reflected wave power of the low frequency case is high as shown in Fig. 5 (c), and the power profile has interference patterns.

Filter thickness dependences of frequency characteristics are summarized in Fig. 6 . In the case of $0 \mathrm{~mm}$ thickness filter, a simple plane is used instead of finite thickness sheet in 3D-modeling. As filter thickness increases, the filter characteristic becomes band pass filter characteristic, i.e., the transmittance becomes worse in the high frequency range. The filter in the range of $0 \mathrm{~mm}-0.5 \mathrm{~mm}$ thickness 


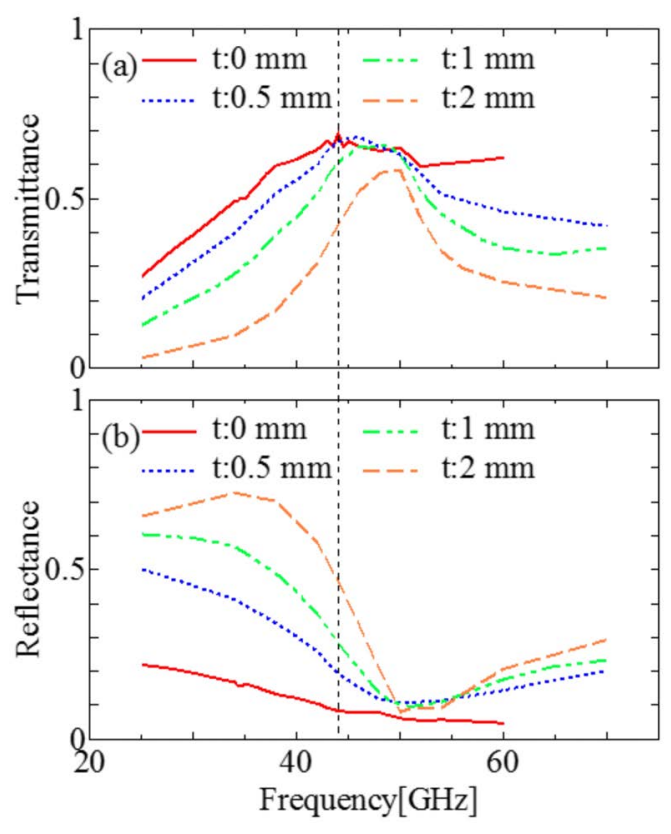

Fig. 6 Filter thickness dependences of frequency characteristics.

shows characteristics of broad-band high pass filter. The sum value of the transmittance and the reflectance is not unity. This is because all transmitted waves and reflected waves do not pass each PDS.

\section{Summary}

We designed broad-band high pass filters using perforated metal sheets with circular holes, and evaluated their characteristics by 3D-numerical analysis. The validity of the numerical analysis is guaranteed by the good agreement between calculated results and measured results. We found that frequency characteristics strongly depend on filter thickness. We concluded that the appropriate filter thickness for ideal broad-band frequency filters is in the range of $0 \mathrm{~mm}-0.5 \mathrm{~mm}$. We will perform characteristic tests of thin filters using numerical analysis and actual measurement.

It also is found that there is an interference pattern in the reflectance power profile, which may blur images of ECE-Imaging diagnostic and inhibit wave guide matching. Therefore, in order to suppress this interference pattern, we will improve our design, for example, of long holes type and of randomly arranged holes type.

\section{Acknowledgement}

This work is performed with the support and under the auspices of the NIFS Collaboration Research program (NIFS15KLEP015).

[1] Y. Nagayama, K. Kawahata, A. England and Y. Ito et al., Rev. Sci. Instrum. 70, 1021 (1999).

[2] H. Tsuchiya et al., Plasma Fusion Res. 6, 2402114 (2011).

[3] Y. Nagayama et al., Rev. Sci. Instrum. 83, 10E305 (2012).

[4] C.-C. Chen, Transmission of Microwave Through Perforated Flat Plates of Finite Thickness, IEEE Trans. Microw. Theory Tech. 21, 1 (1973).

[5] http://www.iti-global.com/

[6] https://www.esi-group.com/ 